EASD

Procedia
EURODYN 2020

XI International Conference on Structural Dynamics M. Papadrakakis, M. Fragiadakis, C. Papadimitriou (eds.) Athens, Greece, 23-26 November 2020

\title{
NUMERICAL AND EXPERIMENTAL STUDY OF A SLENDER CATENARY BRIDGE WITH A NOVEL TENSIONING SYSTEM
}

\author{
Gergely Szabó ${ }^{1}$, Gábor Hochrein ${ }^{2}$ \\ ${ }^{1}$ Bridge design engineer \\ 1031 Budapest, Hungary, Silvanus stny. 45. \\ e-mail:drszabo@pontterv.hu \\ ${ }^{2}$ Bridge design engineer \\ 1119 Budapest, Hungary, Than Karoly u. 3-5 \\ e-mail: hid@pontterv.hu
}

Keywords: catenary bridge, geometrically non-linear dynamics, iso-tensioning

\begin{abstract}
Catenary pedestrian bridges are apparently spreading all over the world. These structures are highly popular as they can form the landscape and provide the pedestrians with excitement and adventure. Consequently, there is a strong need to increase the span length, which might call for the reconsideration of the conventional approaches. A novel concept was proposed with special focus on feasibility and proper static and dynamic performance of slender catenary bridges. A dedicated physical model was built and measured in order to demonstrate the advantages. A long span catenary bridge designed in the framework of a feasibility study was also considered as an ideal candidate for our proposed method.
\end{abstract}

ISSN:2311-9020 C 2020 The Authors. Published by EASD Procedia.

Peer-review under responsibility of the Organizing Committee of EURODYN 2020

doi: $10.47964 / 1120.9167 .20164$ 


\section{INTRODUCTION}

\subsection{Catenary bridge structures}

The main elements of these bridges are the catenary cables that are fixed at both ends. These cables carry the vertical loads, such as the self-weight or ice loading. When necessary, wind cables and connecting cables are also involved in order to provide extra stiffness to the whole structure, which was studied in detail in [1]. The problem with these bridges is that the connecting cables are complicated to tension evenly due to their mutual interaction. There can be loose cables that may vibrate, and the others can be overloaded. On top of all, the overall structural behavior is strongly temperature dependent. The novel approach has been first introduced at the WIBE (World Innovation in Bridge Engineering) prize competition. In this paper a more detailed introduction is addressed.

\subsection{The proposed iso-tensioning system}

To overcome the above mentioned problems, a novel concept is proposed shown in Figure 1. In contrast to conventional catenary bridges, the evenly distributed and temperature independent connecting cable forces can be conveniently ensured by adopting pulleys that are used for railway overhead electric wires for instance. Post-tensioning cables on both sides of the catenary with ballasts at their end points are used to adjust the desired pre-stressing to the structure.

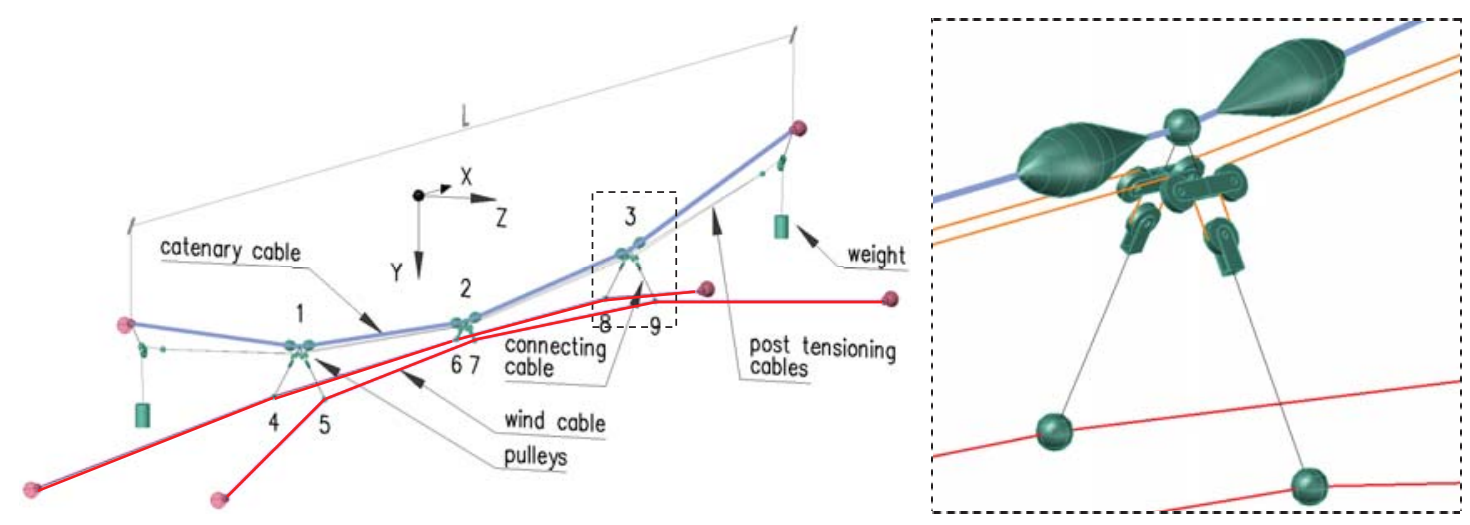

Figure 1: Structural setup of the proposed system.

\section{PRELIMINARY PHYSICAL MODEL}

The structural behavior was investigated by using physical models that are most common in wind engineering [2]. A preliminary model was created in order to qualitatively demonstrate that the proposed system behaved according to our expectations; the connecting cables were indeed evenly pre-stressed, no loose cables were observed. A horizontal load test was also carried out. All the cables were modeled by using fishing line, with lead as weight. The proposed configuration consists of three pulleys in a group belonging to each connecting cable; two of them are joined together and to the catenary, and the third one is fixed to the upper end of the connecting cable. Two post-tensioning cables were driven through the corresponding pulleys with ballast weights at their end points providing the desired internal forces in the connecting cables, resulting in a spatial pre-stressed structure. Although the results were in line with the expectations as to the structural behavior, the unpredictable material of the applied fishing line hindered a precise comparison with numerical results. Therefore, a detailed physical model was constructed based on the experiences gained on this preliminary model. 


\section{DETAILED PHYSICAL MODEL}

\subsection{Model fabrication}

In order to carry out quantitative validation, steel wire ropes were used for the main catenary cable as well as the wind cables. Despite the fact that fishing line found to be improper due to its nonlinear material properties, it was still used for the post-tensioning and the connecting cables with respect to the moderate cable forces in these elements; the strain remained in the linear range. Besides, a multi-strand fishing line is advantageous due to its bending flexibility, which reduces the overall friction of the pulley system. The structure is shown in Figure 2 with coloring the different cable types for better visibility, which will be used later where necessary. The span of the main catenary cable is $\mathrm{L}=14 \mathrm{~m}$. The diameter of the catenary cables is $3.0 \mathrm{~mm}(\mathrm{EA}=560 \mathrm{kN})$, and that of the wind cables is $1.0 \mathrm{~mm}(\mathrm{EA}=75 \mathrm{kN})$. The fishing line diameter of the post-tensioning and the connecting cables is $0.40 \mathrm{~mm}(\mathrm{EA}=2 \mathrm{kN})$. When the bridge was ready and the ballasts were released, the spatial pre-stressed structure was qualitatively studied; the connecting cable forces were indeed close together as desired, loose cables were not found.

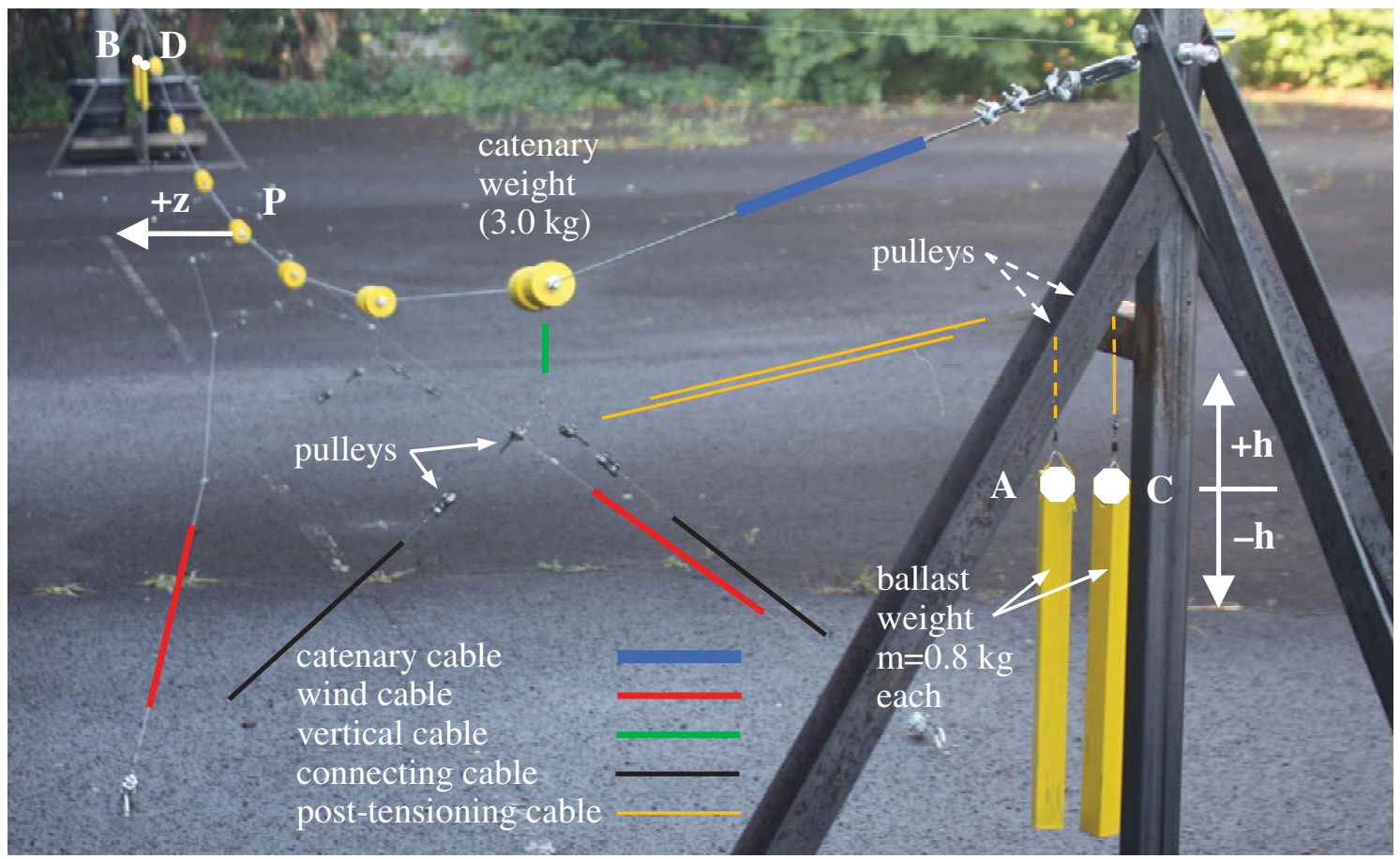

Figure 2. View of the $14 \mathrm{~m}$ long physical model with the monitored points and the colored cable types.

The projected schematic top view of the original and the proposed structural configuration is shown in Figure 3. In case of the conventional structure, the connection between the main catenary cable and the wind cables is made by using connecting cables. The desired internal forces can be adjusted by pre-stressing the wind cables and the connecting cables, which is tedious to carry out precisely due to the mutual interaction of the cables. The connecting cable force distribution depends on the stiffness properties of the whole structure and the pre-stress applied to the cables. The original system is referred to as model v1. 


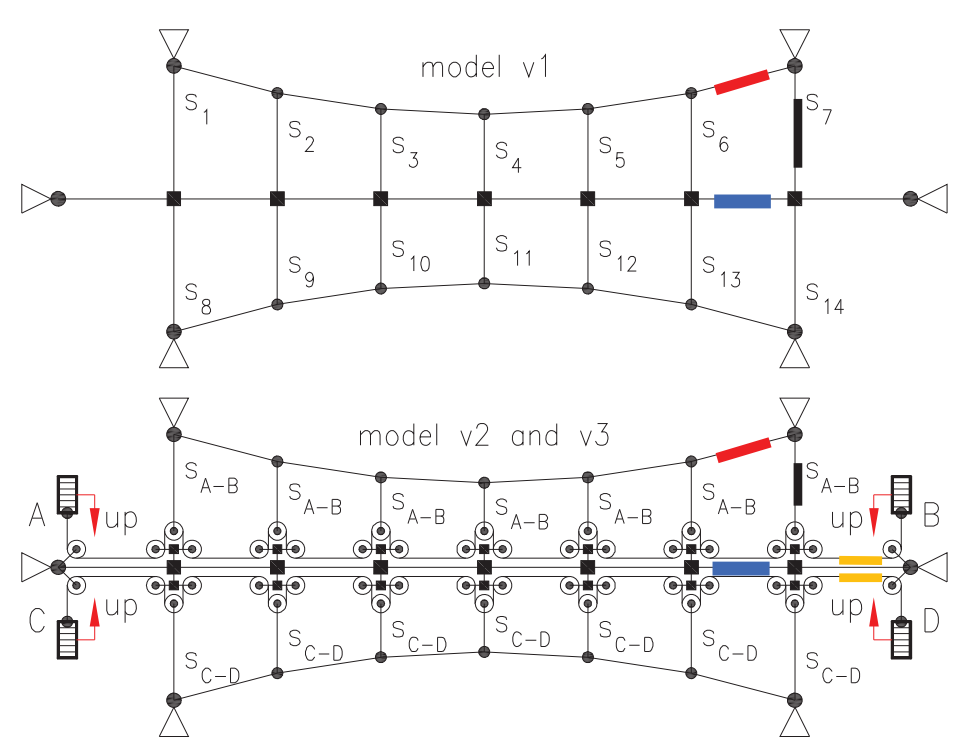

Figure 3: Conventional (top) and the proposed structural system with the connecting cable forces (s).

The proposed system requires ballast weights in order to set the desired cable forces. In contrast to model v1, the connecting cables do not connect the wind cables and the catenary directly, but two post-tensioning cables are included in the whole system belonging to the two wind cables on the two sides of the catenary.

The post-tensioning cables are referred to as A-B and C-D, according to the points on the ballast weights (Figure 3). The whole system of a post-tensioning cable and the belonging connecting cables are referred to as branches A-B and C-D. There are two versions of the proposed system; in case of model v2 all the four ballast weights move independently from each other. Model v3 is different, as the neighboring ballasts are connected together; the motion of ballasts $\mathrm{A}$ and $\mathrm{C}$ is not independent, instead a common upward or downward motion is allowed only. The same applies to ballasts B and D. Model v2 and v3 both produce equal connecting cable forces within a branch if the friction of the pulleys is neglected. For model $\mathrm{v} 2$ the connecting cable forces of the two branches are equal, that is $S_{A-B}=S_{C-D}$.

The pulley system gives an additional flexibility to the system, which may reduce the horizontal stiffness of the main catenary. Therefore, it was of utmost importance to compare the static and dynamic behavior of the proposed models v2 and v3 to the original one of v1. The physical model was constructed with special attention to this need; the pulley system was equipped with a constraint that was capable of fixing the post-tensioning cable, which made it feasible to investigate and compare the behavior of model v1, v2 and v3. The structural details of the physical model are shown in Figure 4.
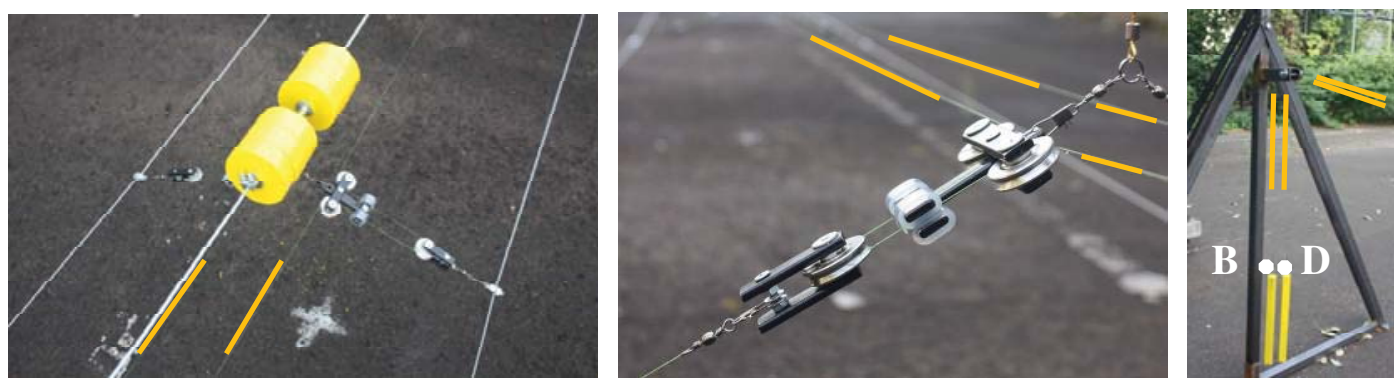

Figure 4: Structural details of the physical model: catenary node, pulleys and ballast weights. 


\subsection{Measurements}

The slender catenary bridges are sensitive to wind loading; therefore the behavior due to horizontal static loading was studied and compared. The catenary nodes were loaded horizontally at each node. The load test can be seen in Figure 5. All the three configurations were tested. Model v1 was set by fixation of the post-tensioning cables around the pulleys. The connecting cable forces are independent from each other in this case. For model v2 the above mentioned fixation was released. Model v3 was set by joining the neighboring ballasts together. The pulley friction can be an important factor in the proposed system; therefore the initial friction value was measured by applying force difference onto the two ends of a test cable hanging on the two sides of a solo V-groove ball bearing. The force difference reached $5 \%$ at the boundary of stall and motion, but drops to 1-2 \% when even moderate motion appears. Time-dependent behavior was also observed, which was explained by the presence of grease in the bearings. Due to the high friction and viscous properties of the pulleys, the whole structure was given slight vibration at each static measurement in order to help the pulleys and the post-tensioning cables arrange and approach to the ideal frictionless state. This was particularly important for model v2. In case of model v1 the ballast weights do not move and there is no pulley rotation either. In case of model v2, on the side the horizontal force pushes the structure toward, the ballasts move downwards as the distance between the catenary and the wind cable decreases. The post-tensioning cable releases ballasts A and B down. On the opposite side, the ballasts $\mathrm{C}$ and $\mathrm{D}$ are lifted upwards. The horizontal displacement of the catenary and the corresponding vertical displacement of ballasts A and C can be seen in Figure 5. In case of model v3 the neighboring ballasts are joined together, therefore no significant ballast motion was observed.
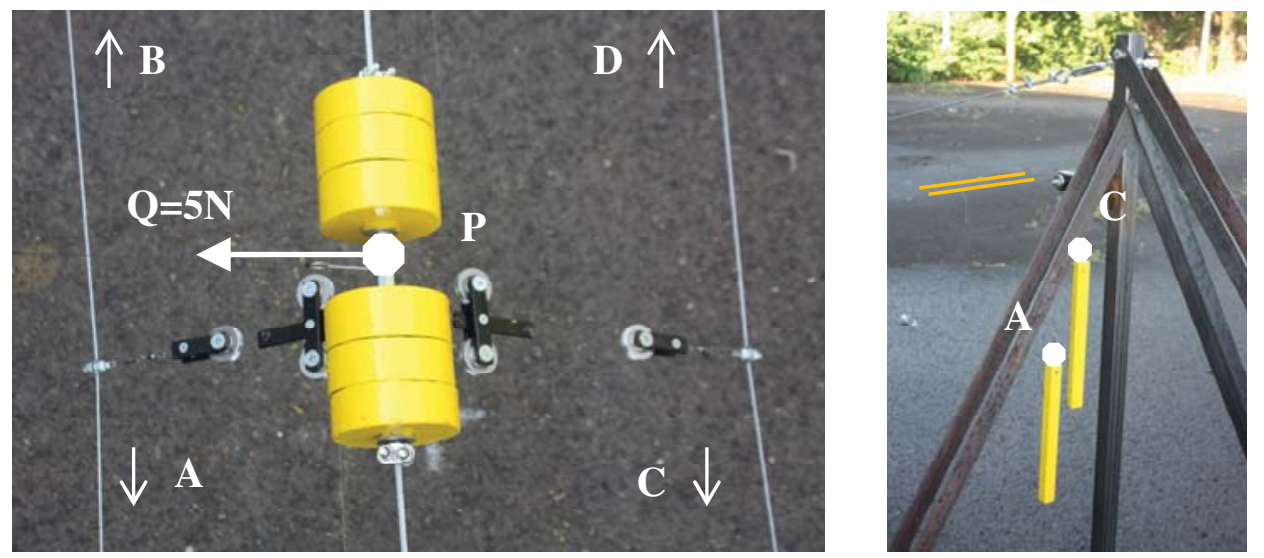

Figure 5: Horizontal static deflection of the catenary and the vertical displacement of the ballasts for model v2.

The dynamic properties were studied by using an accelerometer attached to the middle catenary point $\mathrm{P}$ (see Figure 2 and 5). The first vibration mode of the bridge was considered only, which was excited simply by hand. The symmetrical sway mode was the same for every model. In case of model v2, the sway motion of the catenary was accompanied by the vertical motion of the ballasts with small amplitudes of 1-2 cm only, nevertheless. In case of model v1 and $\mathrm{v} 3$ there were no ballast motion amplitudes observed. 


\section{STATIC NUMERICAL MODEL}

\subsection{Introduction of the numerical approach}

The static behavior was studied on a 72 DOFs numerical model, which was written in Octave (Figure 6). The model consists of two-node spatial truss elements with tension capabilities only. The structural elements are the main catenary cable (blue), the wind cables on both sides (red), the connecting cables (black) and the vertical elements (green). The posttensioning cables and the ballasts are not shown in this model, but their effect is included as described below.

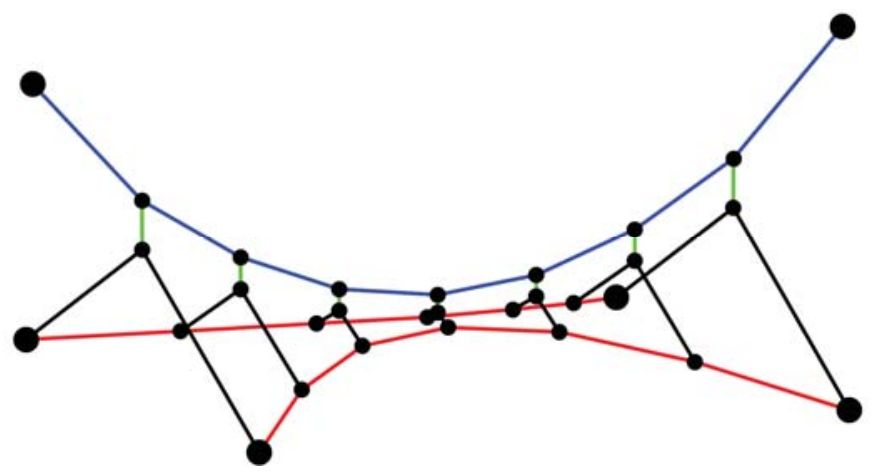

Figure 6: Geometry of the numerical model.

\subsection{Static equations with geometrical non-linearity}

The considered mechanical system involves geometrical non-linearity; therefore the (load control) New-ton-Raphson iterative approach was applied to find static equilibrium. The key step of the process is solving the $\mathbf{K}_{\mathrm{i}}\left(\mathbf{x}_{\mathrm{i}}\right) \Delta \mathbf{x}_{\mathrm{i}}=\Delta \mathbf{q}_{\text {red,i }}$ matrix-equation for the $\Delta \mathbf{x}_{\mathrm{i}}$ displacement error vector due to the reduced force error vector of $\Delta \mathbf{q}_{\mathrm{red}, \mathrm{i}}=-\mathbf{G}_{\mathrm{i}} \mathbf{F}^{\mathbf{- 1}} \mathrm{dt}_{\mathrm{i}}+\Delta \mathbf{q}_{\mathrm{i}}$. The iterative process is to be continued until the $\Delta \mathbf{t}_{\mathbf{i}}=\mathbf{t}-\left(\mathbf{l}_{\mathbf{i}}-\mathbf{l}_{0}-\mathbf{F} \mathbf{s}_{\mathbf{i}}\right)$ and $\Delta \mathbf{q} \mathbf{i}=\mathbf{q}-\left(-\mathbf{G}_{\mathbf{i}} \mathbf{S}_{\mathbf{i}}\right)$ unbalanced kinematic and force vector norms are smaller than a tolerance. In the equations above $\mathbf{x}_{\mathbf{i}}=$ displacement vector of the 24 nodes (see Figure 6 ); $\mathbf{l}_{i}=$ stressed cable length vector; $\mathbf{l}_{0}=$ stress-free cable length vector; $\mathbf{t}=$ kinematic load vector; $\mathbf{q}=$ external force vector; $\mathbf{G}_{\mathrm{i}}=$ geometry matrix containing the direction unit vectors of each cable; $\mathbf{K}_{\mathrm{i}}=$ tangential stiffness matrix; $\mathbf{F}=$ flexibility matrix. All the matrices and vectors are to be updated at each $\mathrm{i}^{\text {th }}$ iteration step. The above detailed Newton-Raphson iteration was carried out in two steps. At the first construction stage the main catenary cable was activated with its vertical loading, which was complemented by the wind cables and the connecting cables at the second stage. Therefore, the sizes of the matrices and vectors are different in these two construction stages. The results of the first stage (e.g. cable length and forces, geometry and stiffness matrices) are used as initial condition for the second one. The first stage is the same for the three models. In case of model v1 the prestressing is defined onto the wind cables and the connecting cables as kinematic loads, i.e. cable shortening. The connecting cable force distribution strongly depends on how the kinematic loads are defined. In case of model v2 and v3, however, the connecting cable forces are the same in every cable and depend on the ballast weight only. The additional horizontal forces are applied at stage two. 


\subsection{Formulation of stiffness matrices}

The key step during the Newton-Raphson process is the formulation of the tangential stiffness matrix that belongs to the actual state of the structure, which can be assembled from the element stiffness matrices. Spatial truss elements were used with an element stiffness matrix:

$$
\mathbf{K}_{\mathrm{e}}=\left[k-\frac{s}{L}\right] \mathbf{e} \cdot \mathbf{e}^{\mathrm{T}}+\frac{s}{L} \mathbf{I}
$$

The spring stiffness is $\mathrm{k}=\mathrm{EA} / \mathrm{L}$, with $\mathrm{EA}=$ normal stiffness; $\mathrm{L}=$ cable length; $\mathbf{e}=$ cable orientation unit vector; s = cable force; $\mathbf{I}=$ identity matrix. The element matrix takes structural and geometrical stiffness into account. For model v1 the formulation of the element matrices is straightforward, the internal force variation of the $\mathrm{j}^{\text {th }}$ connecting cable is $\Delta \mathrm{s}_{\mathrm{j}}=\mathrm{f}\left(\Delta \mathrm{L}_{\mathrm{j}}\right)$, which is the function of the length variation. For model v2 and v3, however, special further considerations have to be made. The force of the $\mathrm{j}^{\text {th }}$ connecting cable depends not only on the displacement of the nodes of its two end points, but of every connecting cable. Therefore, a compatibility equation of the coupled system of the post-tensioning and connecting cables has to be solved at each iteration step in order to obtain the cable force changes based on the displacement variation vector $\Delta \mathrm{x}_{\mathrm{i}}$. The arrangement of branch A-B can be seen in Figure 7.

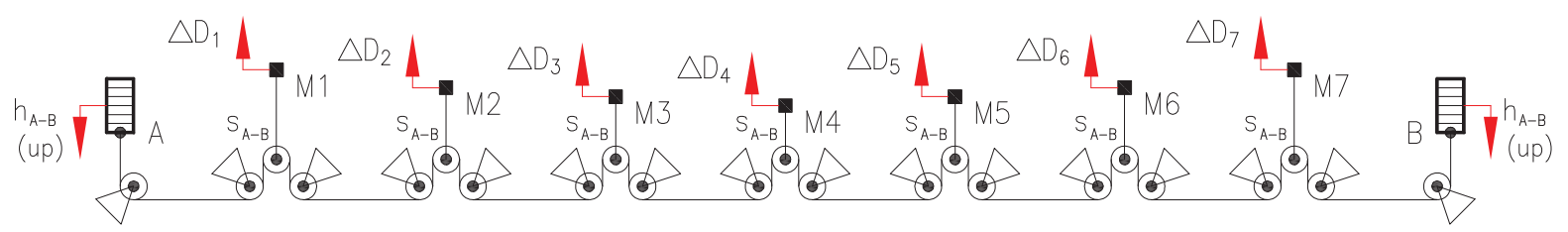

Figure 7: Schematic projected arrangement of branch A-B (D and h are displacement, $\mathrm{s}$ is the cable force).

The forces in the connecting cables of a branch are equal, provided that pulley friction is neglected, which leads to the analogy of serial coupling in electricity. By using this principle, the system shown in Figure 7 was reduced into an inhomogeneous spring, which includes all the connecting cables and the tensioning cable of branch A-B (see Figure 8). The points $\mathrm{M}_{\mathrm{j}}$ were merged into point $\mathrm{M}$, and points $\mathrm{A}$ and $\mathrm{B}$ into $\mathrm{A}-\mathrm{B}$. The total displacement variation onto point $\mathrm{M}$ is calculated as $\Delta \mathrm{x}_{\mathrm{A}-\mathrm{B}}=\Sigma \Delta \mathrm{D}_{\mathrm{j}}$, where $\Delta \mathrm{D}_{\mathrm{j}}=$ end points distance variation (see Figure 8 and 9) of the $\mathrm{j}^{\text {th }}$ connecting cable of branch A-B. The linear reduced stiffness can be calculated by using a reciprocal formula for the serially coupled linear springs of a branch as:

$$
k_{\text {red }, A-B}=\frac{1}{\sum \frac{L_{j}}{E A_{j}}+\frac{L_{A-B}}{4 E A_{A-B}}}
$$

$\mathrm{L}_{\mathrm{j}}=$ length of a connecting cable; $\mathrm{EA}_{\mathrm{j}}=$ normal stiffness of a connecting cable, $\mathrm{L}_{\mathrm{A}-\mathrm{B}}=$ length of the post-tensioning cable, $\mathrm{EA}_{\mathrm{A}-\mathrm{B}}=$ normal stiffness of the post-tensioning cable. Branch C-D can be handled likewise. Symmetrical horizontal loading was considered only.
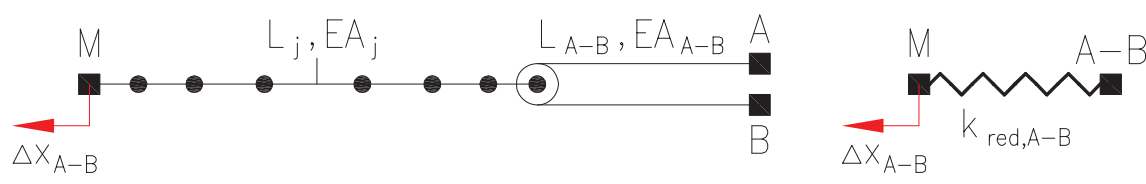

Figure 8: Construction of the idealized spring (left) and its reduced model (right). 

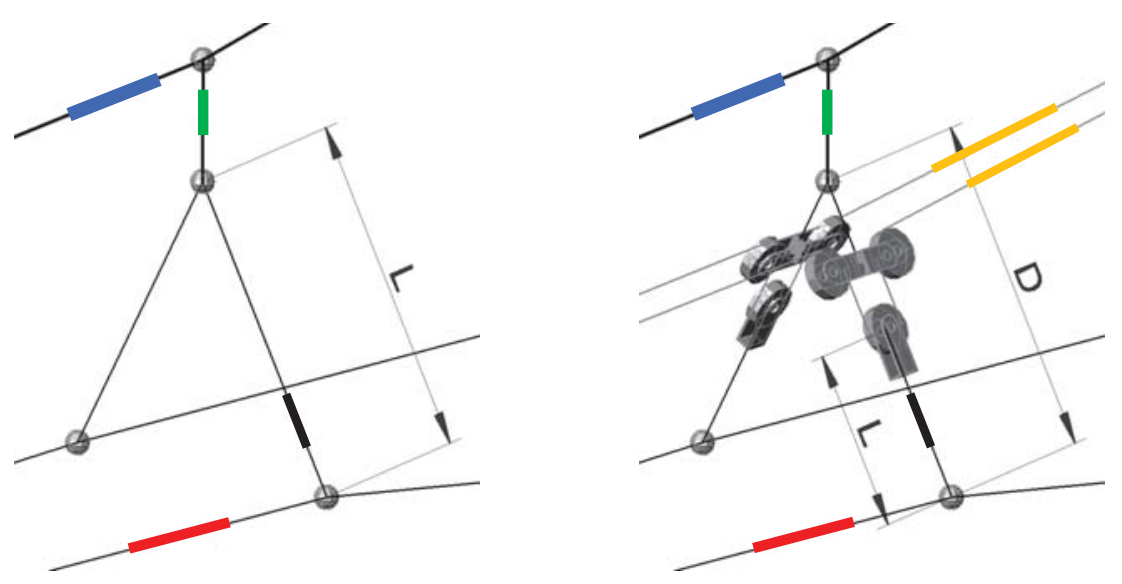

Figure 9: Connecting cables of model v1 (left) and v2-v3 (right) with connecting and post-tensioning cables.

If there is no horizontal load, models $\mathrm{v} 2$ and $\mathrm{v} 3$ are the same as the force is $\mathrm{s}=2 \mathrm{mg}$ in every connecting cable, where $\mathrm{m}=$ weight of one ballast and $\mathrm{g}=9.81 \mathrm{~m} / \mathrm{s}$. The behavior of these two models, however, is different if horizontal load is also applied, which is illustrated in Figure 10. In case of model v2, branches A-B and C-D are independent; therefore the ballast weights can move freely. Consequently the connecting cable forces do not change due to any structural displacement field, that is $\Delta \mathrm{s}_{\mathrm{A}-\mathrm{B}}=0$ and $\Delta \mathrm{s}_{\mathrm{C}-\mathrm{D}}=0$. This can be modeled by simply eliminating term $\mathrm{k}$ in the element stiffness matrix of the connecting cables (1), which means that the geometrical stiffness belonging to the constant connecting cable forces exists only. It should be noted, however, that instead of using zero value for $\mathrm{k}$, a small nonzero value is better to use for faster convergence. In case of model $\mathrm{v} 3$ points $\mathrm{A}-\mathrm{C}$ and $\mathrm{B}-\mathrm{D}$ are connected together; the two inhomogeneous springs belonging to the branches A-B and C-D are now coupled in a parallel way.
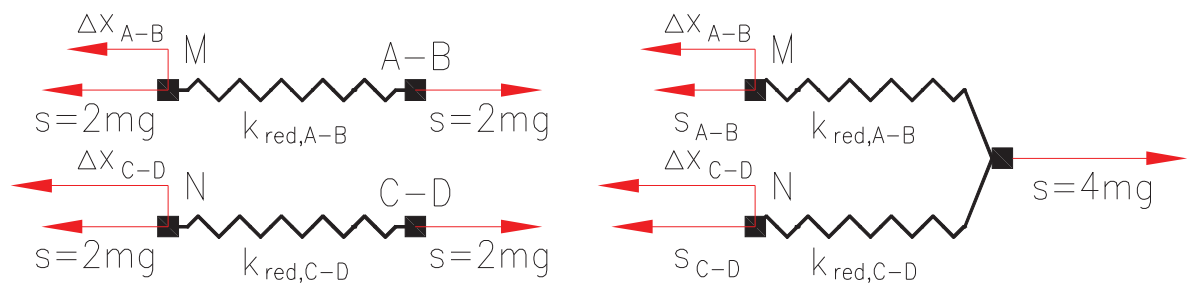

Figure 10: Difference between the behavior of model v2 (left) and v3 (right).

The internal force variation for branch A-B is $\Delta s_{A-B}=f\left(\Delta D_{j}, j=1 . .2 n\right)$ and that for the branch $\mathrm{C}-\mathrm{D}$ is $\Delta \mathrm{s}_{\mathrm{C}-\mathrm{D}}=\mathrm{g}\left(\Delta \mathrm{D}_{\mathrm{j}}, \mathrm{j}=1 . .2 \mathrm{n}\right)$, where $\mathrm{n}=$ number of the connecting cables on one side, namely 7 in our case. Unlike model v2, the connecting cable forces are not invariant of the reduced displacement variation in case of model v3. If the reduced displacement variation of $\Delta \mathrm{x}_{\mathrm{A}-\mathrm{B}}$ and $\Delta \mathrm{x}_{\mathrm{C}-\mathrm{D}}$ are not equal, a difference will appear between the connecting cable forces of the two branches.

The reduced displacement variations for branch $\mathrm{A}-\mathrm{B}$ is $\Delta \mathrm{x}_{\text {red,A-B }}=\Delta \mathrm{x}_{\mathrm{A}-\mathrm{B}}-\left(\Delta \mathrm{x}_{\mathrm{A}-\mathrm{B}}+\Delta \mathrm{x}_{\mathrm{C}-\mathrm{D}}\right) / 2$ and for branch $\mathrm{C}-\mathrm{D}$ is $\Delta \mathrm{x}_{\mathrm{red}, \mathrm{C}-\mathrm{D}}=\Delta \mathrm{x}_{\mathrm{C}-\mathrm{D}-}-\left(\Delta \mathrm{x}_{\mathrm{A}-\mathrm{B}}+\Delta \mathrm{x}_{\mathrm{C}-\mathrm{D}}\right) / 2$. If symmetrical horizontal forces are applied, the connecting cable force variations is be calculated as $\Delta \mathrm{s}_{\mathrm{A}-\mathrm{B}}=\Delta \mathrm{x}_{\text {red,A-B }} \cdot \mathrm{k}_{\text {red,A-B }}$ and $\Delta \mathrm{s}_{\mathrm{C}-\mathrm{D}}=\Delta \mathrm{x}_{\text {red,C-D }} \cdot \mathrm{k}_{\text {red,C-D. }}$. Compared to model $\mathrm{v} 2$, stiffness $\mathrm{k}$ in the element stiffness matrix is not zero. The normal stiffness $k$ for each connecting cable of branch A-B and C-D were selected as $n \cdot k_{\text {red,A-B }}$ and $n \cdot k_{\text {red,C-D }}$, respectively. This intuitive stiffness approximation of the connecting cables gave fast convergence in case of symmetrical total horizontal loading. 


\section{DYNAMIC NUMERICAL MODEL}

The dynamic behavior of the three models was studied by extending the static equations with the inertia and damping forces. A non-iterative incremental time advancement version of leap-frog scheme was used. Also, a nested Newton-Raphson scheme can also be developed.

Model v1 required to solve Equation (3):

$$
\mathbf{M} \Delta \mathbf{a}_{\mathrm{t}}+\mathbf{C} \Delta \mathbf{v}_{\mathrm{t}}+\mathbf{K}_{\mathrm{t}}\left(\mathbf{x}_{\mathrm{t}}\right) \Delta \mathbf{x}_{\mathrm{t}}=\Delta \mathbf{Q}_{\mathrm{t}}
$$

In (3) $\mathbf{M}=$ mass matrix; $\mathbf{C}=$ damping matrix; $\mathbf{a}=$ acceleration vector; $\mathbf{v}=$ velocity vector; $\mathbf{x}=$ displacement vector; $\mathbf{Q}=$ external load vector. As the structure is geometrically non-linear, the stiffness matrix had to be updated at each time step based on the geometrical position of the nodes (see Figure 6) and the cable forces. Although the explicit scheme was easy to implement, the time step for a stable and accurate solution was typically 10 times less than usual in linear dynamics.

Model v2 required the modification of the equation (3) into (4):

$$
\mathbf{M} \Delta \mathbf{a}_{\mathrm{t}}+\mathbf{C} \Delta \mathbf{v}_{\mathrm{t}}+\mathbf{K}_{\mathrm{t}}\left(\mathbf{x}_{\mathrm{t}}\right) \Delta \mathbf{x}_{\mathrm{t}}+\Delta \mathbf{P}\left(\mathrm{a}_{\mathrm{t}}, \mathrm{v}_{\mathrm{t}}\right)=\Delta \mathbf{Q}_{\mathrm{t}}
$$

The elements of vector $\Delta \mathbf{P}_{\mathrm{t}}$ are determined by solving $\Delta \mathbf{P}_{\mathrm{t}}=\mathbf{G}_{\mathrm{t}} \Delta \mathbf{s}_{\mathrm{t}}$, where $\Delta \mathbf{s}_{\mathrm{t}}=$ internal force vector increment belonging to the connecting cables. Unlike static behavior, the connecting cable forces do change due to inertia and damping forces accompanied by the ballast weight motion, therefore $\Delta \mathbf{P}_{\mathrm{t}}$ is the function of $\mathbf{a}_{\mathrm{t}}$ and $\mathbf{v}_{\mathrm{t}}$ of the structural nodes. In Figure 11 the coupled system of the post-tensioning cable and the connecting cables is shown for model v2, which was reduced into an idealized spring system. The two ballasts of a branch move identically due to symmetrical horizontal loading. The spring representing branch A-B is excited at point $\mathrm{M}$ with displacement increment of $\Delta \mathrm{x}_{\mathrm{A}-\mathrm{B}}=\Sigma \Delta \mathrm{D}_{\mathrm{j}}$, where $\mathrm{D}$ is illustrated in Figure 9. The spring of branch C-D is derived likewise. In order to improve the performance of model v2, each ballast can be equipped with a linear hydraulic damper (c) and a nonlinear spring that is inactive until a prescribed displacement $(\delta)$ is reached. The dynamic equation of motion is solved for the vertical ballast displacement variation $\Delta \mathrm{h}$, from which the cable force change in the reduced spring of a branch is $\Delta s=(\Delta \mathrm{x}-\Delta \mathrm{h}) \cdot \mathrm{k}_{\mathrm{red}}$, which is equal to the connecting cable force increments of the same branch. Based on the connecting cable force increments, $\Delta \mathbf{P}_{\mathrm{t}}$ can be compiled, and the displacement, velocity and acceleration increments of the 24 structural nodes and the four ballasts can be calculated at a time step.

Model v3 is simpler than v2 in case of horizontal loading, as the vertical motion of the ballast and the consequent dynamic forces can be neglected. Instead, the principles introduced at the static numerical modeling apply here. The difference is that the internal forces of the connecting cables are taken into consideration in the $\Delta \mathbf{P}_{\mathrm{t}}$ vector rather than in the term $\mathbf{K}_{\mathrm{t}} \Delta \mathbf{x}_{\mathrm{t}}$, which means that the internal forces are handled as external loadings. This is important with respect to the explicit time advancement scheme as there is no internal iteration within a time step. The nonlinear dynamic calculation was tested by applying quasi-static test loading; the results were in good agreement with that of the nonlinear static calculations for all the three models.
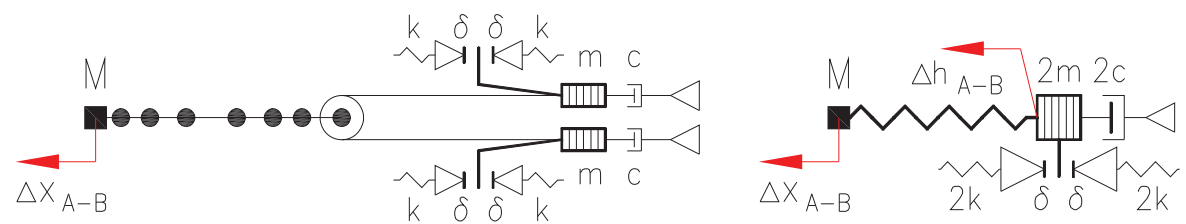

Figure 11: Dynamic model of the idealized spring for branch A-B (left) and its reduced model (right). 


\section{VALIDATION}

In the previous chapters the proposed structural system was investigated by means of physical and numerical approaches. The input for the numerical simulation was set in accordance with the measured physical model; the normal stiffness of the steel wires and the multi-strand fishing line were measured through tensional tests. The weight of each structural part was also determined. Neither hydraulic damping, nor nonlinear springs were applied to the ballasts. A horizontal static load of $5 \mathrm{~N}$ was applied to the catenary nodes. The horizontal displacement showed linear tendency for every model for this load. In Table 1 the measured and calculated horizontal displacement in mm of point $\mathrm{P}$ (see Figure 12) and vertical displacement of ballast points $\mathrm{A}$ and $\mathrm{C}$ are compared. The measured and simulated displacement values are in good agreement for model $v 1$ and $v 3$. The results of model $v 1$ and $v 3$ are close together, showing that there is no significant loss in the overall stiffness due to the dominant horizontal loading by applying the proposed post-tensioning system of model v3. Model v2, however, gave 2-3 times larger displacement, which can be explained by the unconstrained vertical displacement of the ballasts and the consequently constant connecting cable forces. The connecting cables with constant forces need to change spatial orientation in order to balance the horizontal load, which is the source of the significantly larger horizontal displacement. The measured horizontal displacement is lower by 35 percent than the calculated one due to the friction of the pulleys. The ballast displacement shows the difference between models v2 and v3; points A and $\mathrm{C}$ move in the opposite direction and so do points $\mathrm{B}$ and $\mathrm{D}$ in case of model v2. On the other hand, in case of model v3, points A-C and B-D are connected together, resulting in a small common displacement, which constrains the horizontal displacement of the catenary.

\begin{tabular}{lllllll}
\hline Models & & Physical & & \multicolumn{3}{c}{ Numerical } \\
& P_hor & A_vert & C_vert & P_hor & A_vert & C_vert \\
\hline v1 & 21 & - & - & 22 & - & - \\
v2 & 50 & -190 & 178 & 67 & -276 & 276 \\
v3 & 28 & 3 & 3 & 29 & 8 & 8 \\
\hline
\end{tabular}

Table 1: Horizontal displacement of point $\mathrm{P}$ and vertical displacement of points $\mathrm{A}$ and $\mathrm{C}[\mathrm{mm}]$.

The developed dynamic numerical method was also validated. The natural frequencies were determined by FFT analysis of the time series of the geometrically non-linear time stepping method. The simulation was started on the completed bridge structure as a result of the nonlinear static calculation. The time dependent horizontal load was applied to each catenary node; it was ramped up in a quasi-steady manner and then set to zero in one time step, obtaining a free decay oscillation to study.

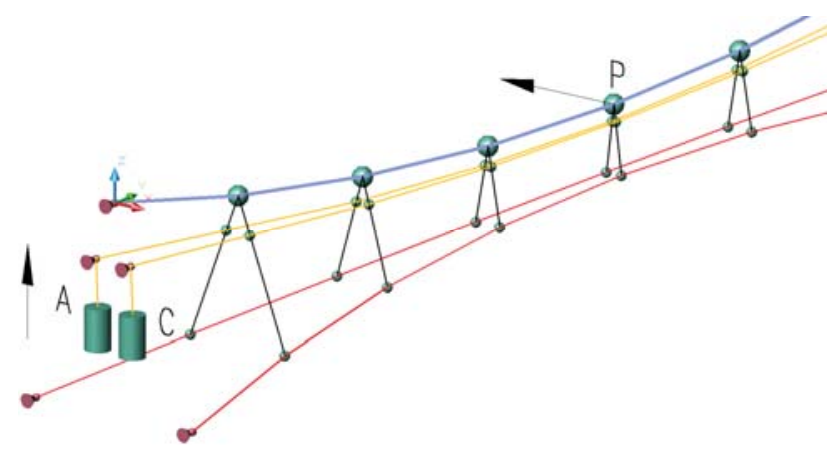

Figure 12: Investigated points and displacement directions. 
The results are shown in Table 2 for the three models and with stage 1 for the catenary alone state. The measured and simulated natural frequencies of the sway mode are in good agreement. According to expectations, model v1 has the highest frequency as it has the highest stiffness. Model v3 showed higher static deflection and the frequency is consequently lower. Model v2 is interesting as the sway mode has a frequency close to that of models v1 and v3. On the other hand, the extra freedom of the ballasts provided a slow vibration mode that belongs to the vertical motion of the ballasts and the horizontal motion of the catenary. The frequency is around $0.30 \mathrm{~Hz}$, which is shown in Table 2 in parentheses. This mode was spotted numerically but did not show up in the measurements, which is explained by the behavior of the pulleys; the bearings of this size give relatively high friction and viscosity, which might hinder the whole pulley system from accommodating this vibration mode.

\begin{tabular}{lll}
\hline Models & Physical & Numerical \\
\hline stage1 & 0.68 & 0.66 \\
v1 & 1.50 & 1.44 \\
v2 & 1.37 & $1.30(0.30)$ \\
v3 & 1.38 & 1.28 \\
\hline
\end{tabular}

Table 2: Natural frequencies of the symmetrical sway mode.

\section{INDUSTRIAL APPLICATION}

\subsection{The considered long-span catenary bridge}

In this chapter an $\mathrm{L}=700 \mathrm{~m}$ main span catenary is considered that has been designed by Pont-TERV Ltd in the framework of a feasibility study in 2017 (see Figure 13). The bridge geometry is similar to the physical model that was studied earlier. The proposed novel tensioning system and the adequate numerical approach were adopted to this bridge structure. It is highlighted that this span length maximization is under research in case of road bridges [4].

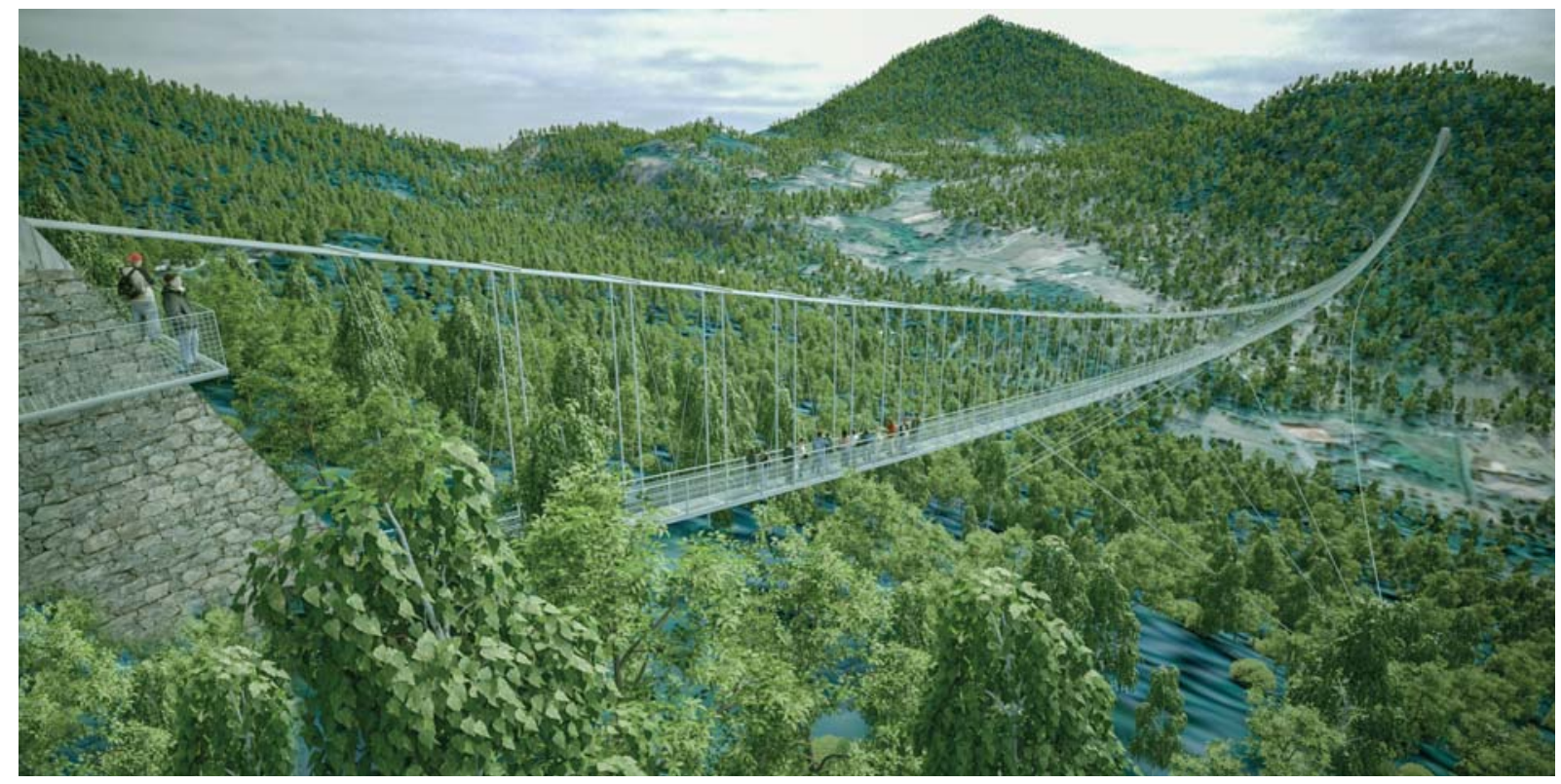

Figure 13: The considered long span catenary structure (Feasibility study, courtesy of Pont-TERV Ltd, 2017). 


\subsection{Application of the proposed iso-tensioning system}

At first, the horizontal displacement of the structure due to horizontal loading on the catenary is illustrated in Figure 14. It can be seen that the catenary together with the wind cables moves sideways.

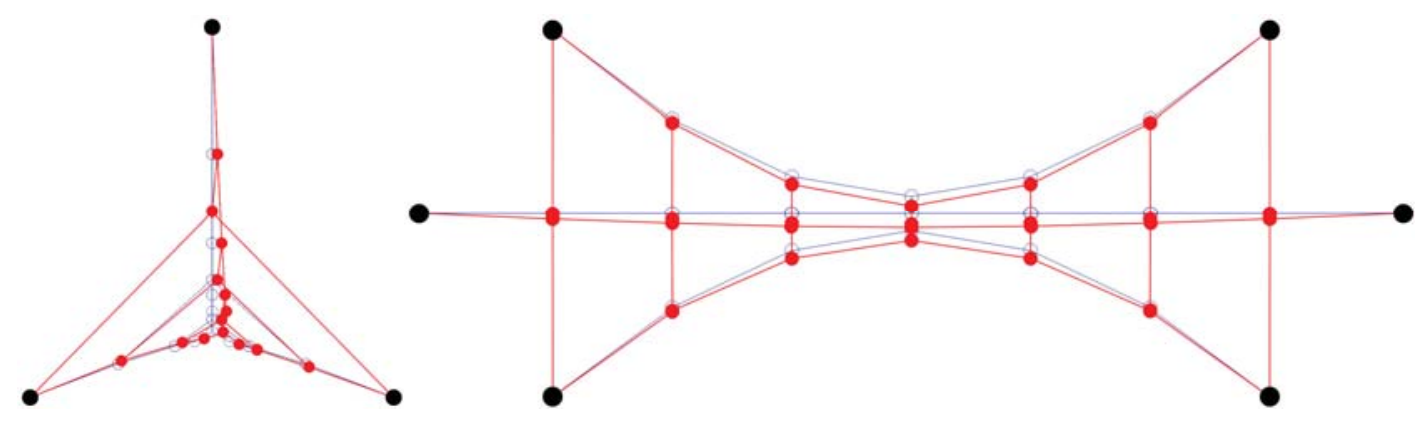

Figure 14: Horizontal deflection of the catenary.

The selected ballast weight is $4 \mathrm{t}$ each. The static test loading was horizontal $\mathrm{Q}_{\text {top }}=14 \mathrm{kN}$ at the nodes of the catenary, and $\mathrm{Q}_{\text {bottom }}=31 \mathrm{kN}$ at the nodes representing the bridge walkway. The horizontal loading was ramped up within 20 seconds and then was kept constant. The horizontal displacement of point P for models v1, v2 and v3 is shown in Figure 15. Both models are common in reach a horizontal displacement around $60-80 \mathrm{~cm}$ when the ramped load reaches its maximum value. This displacement belongs to the spatial deformation of the whole bridge structure (see Figure 14).

The displacement values remain constant except for model v2, which shows increasing tendency with time. This creep-like behavior is due to the ballast motion pulled by the posttensioning cables, which is explained in Figure 15 (right hand side); $\mathrm{z}_{0}$ is the deflection due to short-term load, $\mathrm{z}_{\mathrm{t}}$ is the long-term deflection. The displacement growth speed can be decreased by involving dampers shown in Figure 11. In our calculations a value of $c=10 \mathrm{kNs} / \mathrm{m}$ was selected. The maximum displacement of model v2 was reached when the orientation of the connecting cables changed in order to reach equilibrium. It is important to note that there is a limit for the static horizontal loads to be balanced by the connecting cables, as the forces in these cables are constant under any external load conditions. To overcome this problem, the ballast motion was constrained by using supports that were activated after a prescribed vertical motion (h), which restricted the horizontal displacement of the catenary as well. At each ballast, a spring with $\mathrm{k}=10000 \mathrm{kN} / \mathrm{m}$ was defined that was activated when the ballast vertical displacement exceeded $\delta=2.00 \mathrm{~m}$. With this non-linear constraint the creep-like behavior could be well controlled. In Figure 15 (left hand side), the results of model v2 both with and without constraint are shown (red and green curves).
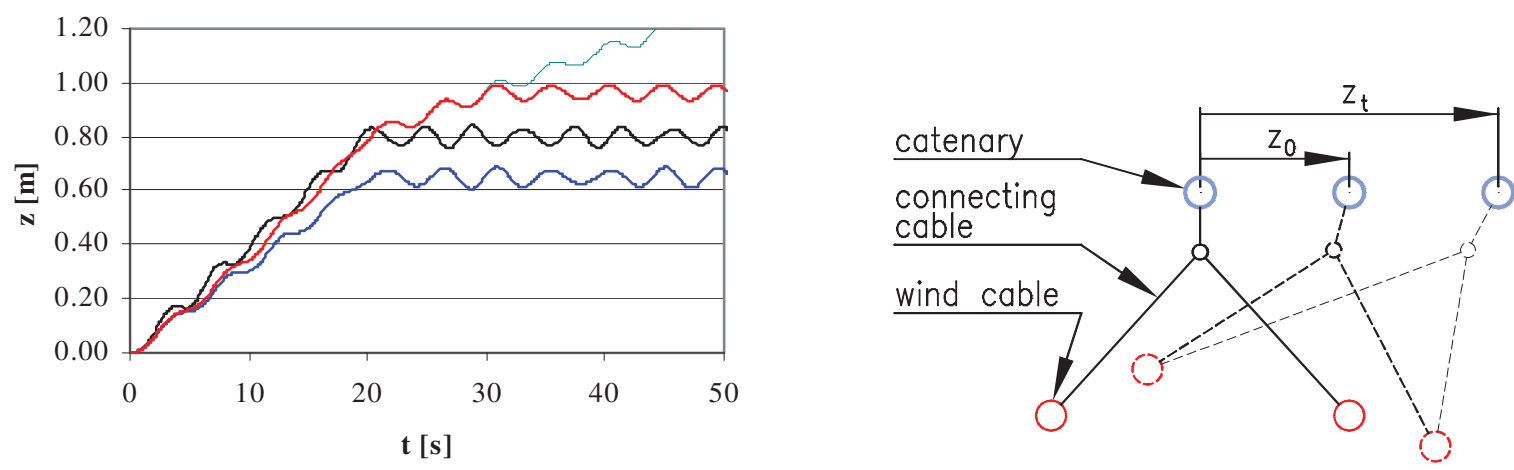

Figure 15: Horizontal displacement due to static load (v1: black, v2-constrained: red, v2: green, v3: blue). 
In Figure 16 the dynamic performance of the three models are compared. The model v2 is investigated with the above mentioned constraint, the no-constrain condition is not shown now. The test load is the same as the quasi-static one, but when its maximum was reached, the load was dropped to zero in one time step, leading to a free decay oscillation. The results of model v1 and v3 are close together. Model v2 shows larger damping, which can be explained by the damped motion of the ballasts; the horizontal displacement of the walkway are transferred by the post-tensioning cables to the ballasts that can move freely making the dampers work. The neighboring ballasts are prevented from moving in the opposite direction in case of model v3; therefore the hydraulic dampers do not increase the overall damping at all.

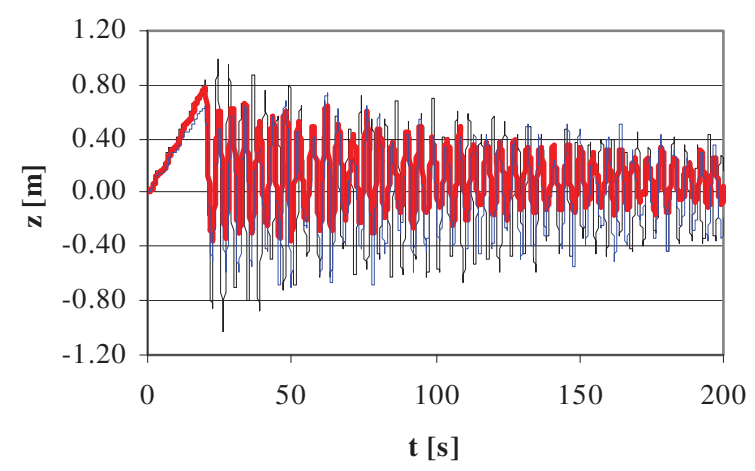

Figure 16: Horizontal displacement due to sudden load (v1: black, v2-constrained: red, v3: blue).

Having studied the structural behavior of the three different models due to ideal loads, a real test was also carried out. An along-wind time series was generated based on the turbulent spectrum proposed by the Eurocode. By using the wind speed values at each time step, the loading to the catenary and walkway nodes was determined. Unlike nonlinear aerodynamic load models, a simplified along-wind loading of $\mathrm{q}_{\mathrm{x}}=0.5 \rho \mathrm{U}^{2} \mathrm{c}_{\mathrm{x}} \mathrm{A}$ was chosen, where $\rho=$ air density; $U$ = wind velocity; $c_{x}=$ static force coefficient of the certain structural element; $A=$ reference area. The effect of the displacement and velocity of the bridge on the aerodynamic forces is neglected. The structural logarithmic damping value of $\delta=0.22$ was selected as a sum of the inherent structural damping $(\delta=0.02)$ and the aero-dynamic damping.

All the parameters were chosen in accordance with Eurocode (terrain category: III, height over ground: $80 \mathrm{~m}$ ). Due to the high reduced wind speed, the simplified aerodynamic load model is justified; therefore advanced three-dimensional fluid-structure interaction model [2] can be omitted for this problem. The results are shown in Figure 17. For model v2 each ballast was equipped with a hydraulic damper and a non-linear spring that was introduced earlier. The dynamic displacement values of model v1 and v3 show the same tendency, but that is apparently lower in case of model v 2 due to the additional effect of the dampers of the ballasts.

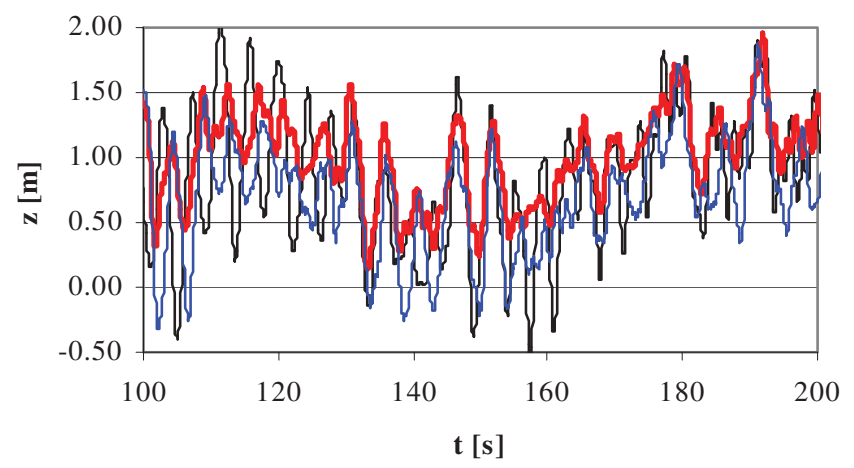

Figure 17: Horizontal displacement of point P due to buffeting (v1: black, v2-constrained: red, v3: blue). 


\section{CONCLUSIONS}

- In this paper a novel post-tensioning method was proposed for long span catenary bridges. The system involves pulleys that has a number of advantages. First of all, the prestressing is temperature-independent. Seconly, the connecting cable forces are evenly distributed, therefore any vibration of a loose cable is unlikely. The proposed structural configuration was tested on a physical model. The evenly distributed connecting cable forces were indeed observed, which was our main goal to achieve. An adequate numerical model was also developed. Three configurations were investigated; a conventional one named model $\mathrm{v} 1$, and the novel pulley based system with and without fixing the neighboring ballasts together, which are models v3 and v2, respectively. The measured and calculated results were in good agreement.

- The friction of the pulleys were not considered in the numerical models. Their effect will be studied in our advanced models in the future.

- The validated numerical model was tested on a catenary bridge of real sizes as well. It was found that model v2 and v3 worked properly due to buffeting forces, as the dynamic responses did not exceed that of model v1, which indicated that the loss in the horizontal stiffness of the proposed systems can be avoided. The novel configuration v2 and v3 are basically different in nature; v3 is simpler as the neighboring ballasts are connected together, which results in a stiffness close to model $\mathrm{v} 1$ and a consequently similar static and dynamic behavior. The more sophisticated v2, however, requires special treatment in order to preserve stiffness; a constraint is necessary to the ballasts that controls the displacement. On the other hand, applying hydraulic dampers too, the overall damping of the bridge can be increased. The choice between model v2 and v3 therefore requires profound design and economic considerations.

- To conclude, the main goal of a temperature-independent and evenly distributed connecting cable forces has been achieved by the proposed structural configuration(s) with at least equivalent static and dynamic performance compared to the conventional catenary bridges. The additional damping experienced in case of model $\mathrm{v} 2$ is beyond our expectations, which will be further investigated as it can be essential in span length maximization.

\section{REFERENCES}

[1] M.H. Huang, D.P. Thambiratnam, N.J. Perera, Vibration Characteristics of Shallow Suspension Bridger with Pre-tensioned Cables, Engineering Structures 27(8), 12201233, 2005.

[2] S. Stoyanoff, P. Irwin, Flutter Analysis of Lions' Gate Bridge during Deck Replacement, Proceedings of the $6^{\text {th }}$ Asia-Pacific Conference on Wind Engineering, 2005.

[3] G. Szabó, J. Györgyi, G. Kristóf, Advanced flutter simulation of flexible bridge decks, Coupled Systems Mechanics 1(2), 133-154, 2012.

[4] Y.J. Ge, Aerodynamic challenge and limitation in long-span cable-supported bridges, Proceedings of Advances in Civil, Environmental, and Materials Research, 2016. 